Cite this: RSC Adv., 2014, 4, 14569

\title{
In situ microspectroscopic monitoring within a microfluidic reactor $\dagger$
}

Received 25th February 2014

Accepted 5th March 2014

\author{
Robert Zmijan,,$^{a}$ Michele Carboni, $\neq^{a b}$ Lorenzo Capretto, ${ }^{a}$ Eugen Stulz ${ }^{\star b}$ \\ and Xunli Zhang*a
}

DOI: $10.1039 / c 4 r a 01650 e$

www.rsc.org/advances

A novel in situ microspectroscopic monitoring system was developed, coupling a microspectrophotometer with a microscope and optical elements, for real time monitoring of chemical reaction progress within a microfluidic reactor by absorbance measurements. The formation process and rapid changes in size and shape of silver nanoprisms were successfully detected and reveal that these changes take place in a very short section of the microreactor.

In this communication we present a novel microspectroscopic monitoring system as a fast and low-cost analytical tool for in situ monitoring of rapid (millisecond) changes in chemical reactions. Optical monitoring of fast evolution of chemical reactions with conventional bench spectrophotometers is not always possible due to the long acquisition time required. In addition, the simultaneous presence of species at different stages of reactions within the same batch can lead to broad and overlaid spectra. With recent advances in microfluidic technology, microreactors have proven to be an excellent platform not only for carrying out chemical reactions, but also for real time monitoring of reaction progress in order to elucidate the reaction mechanism and kinetics. As reactions evolve along the whole microchannel length, all the chemical species are present simultaneously at different points of the channel allowing detection of reaction progress in a spatially-resolved format. In particular for the formation of nanoparticles, changes in optical properties of the reaction system can act as direct indicators for the transition from seeds to the final desired nanoparticles. ${ }^{\mathbf{1}}$ This could be used to better understand the formation process, which is crucial for controlled production of nanoparticles with reproducibility. In this regard, spatially-resolved kinetic studies

${ }^{a}$ Engineering and the Environment, University of Southampton, Southampton SO17 1BJ, UK. E-mail: XL.Zhang@soton.ac.uk; Tel: +44 (0)2380595099

${ }^{b}$ School of Chemistry and Institute for Life Sciences, University of Southampton, Southampton SO17 1BJ, UK. E-mail: est@soton.ac.uk; Tel: +44 (0)23 80599369

† Electronic supplementary information (ESI) available: Details of the instrumental setup and analytical data. See DOI: 10.1039/c4ra01650e

\$ These authors contributed equally to the work. have been conducted by collecting photoluminescence images and emission spectra during the formation of cysteine-capped CdS quantum dot nanocrystals within a microfluidic reactor. ${ }^{2}$ However, this method is not suitable for non-fluorescent metal nanoparticles where absorbance measurements are commonly used. Furthermore, due to the resulting short optical path length of microchannels, this method becomes problematic where low absorbances are to be detected. Although successful attempts have been made to increase the optical path length, e.g. by increasing microchannel dimension for a selected section, or adding optical elements for multi-pass spectroscopy, ${ }^{3}$ they generally limit the detection to specific points of the channel.

We have now developed a novel absorbance detection system based on an inverted microscope coupled with a UV-Vis-NIR micro-spectrophotometer for spatially-resolved spectroscopy measurements at any point of interest along the microfluidic channel. A schematic of the experimental setup is shown in Fig. 1a.

In the optical setup, we used a $10 \mathrm{~W}$ tungsten halogen lamp as light source linked through an optical fibre (i.d. $600 \mu \mathrm{m})$ to an inlet tube which held a collimating lens (focal length $25 \mathrm{~mm}$ ) and an aspherical achromatic lens (focal length $40 \mathrm{~mm}$ ). This setup allowed us to focus the divergent light from the inlet tube to pass the microfluidic channel at its thinnest beam waist. The size of the light spot $(250 \mu \mathrm{m})$ compares well with the width of the microfluidic channel $(210 \mu \mathrm{m}$, Fig. 1b) which grants an excellent space resolution, and the spot can be moved precisely to the selected point of interest along the microchannel with the $X-Y$ microscope stage. For the precise light delivery to the detector, the microscope condenser lens holder was replaced with a custom assembly that held the inlet tube in alignment with the optical axis of the microscope objective. Another assembly of lenses was connected to the side port of the microscope to focus the light into the optical fibre (see Fig. S1 of $\mathrm{ESI} \uparrow$ for details of the instrument setup).

The microfluidic reactor (Fig. 1c) was made of poly (methyl methacrylate) (PMMA) having 3 inlets and a main reaction channel of $\sim 108 \mathrm{~cm}$ in total length, and having a rectangular cross-section of width $\times$ depth $=210 \times 300 \mu \mathrm{m}$. The microreactor 
(a)

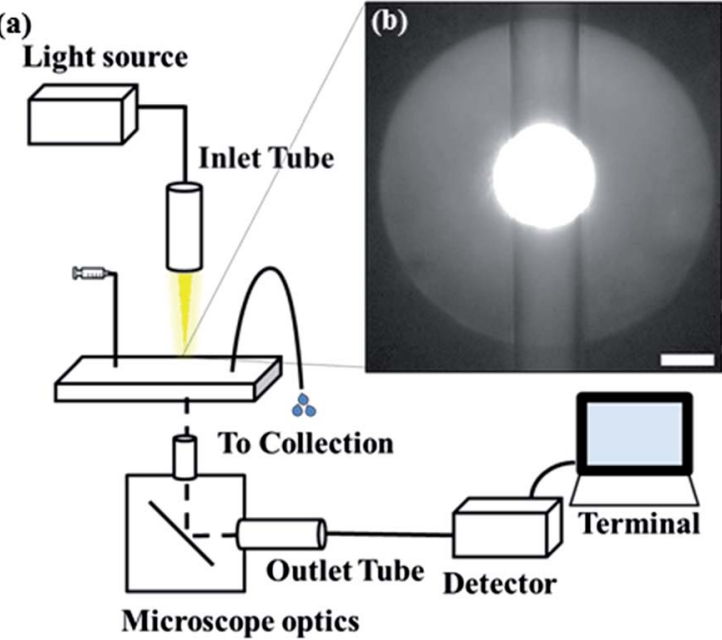

(c)

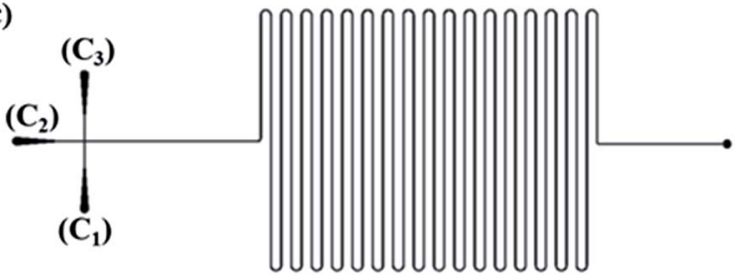

Fig. 1 (a) Schematic layout of the experimental setup, (b) focused light spot on the microfluidic reactor channel (scale bar: $200 \mu \mathrm{m})$, and (c) the microfluidic reactor layout where $C_{1}, C_{2}$ and $C_{3}$ are three channel inlets.

was placed on the microscope stage and the light source holder height was adjusted to launch the beam waist at its thinnest crosssection through the centre of the channel. A $60 \times$ microscope objective was used to focus and collect the maximum of the light passing through the microfluidic channel. A custom assembly of lenses and a sub multi assembly (SMA) fibre coupler were fixed in a concentric outlet tube which was attached to the side port through a C-mount adapter and directly connected to the spectroscopy detector with an optical fibre. With this setup we reached a minimum integration time of $1.05 \mathrm{~ms}$ which was the lower limit of the detector but sufficient for the light source to almost reach the saturation limit with the build-in zoom of the microscope $(1.6 \times)$ and the $60 \times$ objective. For absorbance measurements, 100 scans were taken and averaged for each measurement in order to reduce the background noise. We also collected the background at different points along the channel with water flowing through the reactor, and averaged the spectra in order to obtain a baseline valid for the entire length of the channel.

We validated the developed system against a conventional bench spectrophotometer by measuring the absorbance of solutions at known concentration levels. Fig. 2 shows samples of spectra collected from aqueous tartrazine solutions in a concentration range of $20-100 \mu \mathrm{M}$ passing through the microreactor channel. For comparison, spectra collected from samples with the same concentrations using the benchtop spectrometer are also displayed. As can be seen from Fig. 2a, the spectra collected from the microreactor match very well with that from the spectrometer cuvette; all spectra exhibit a maximum absorbance peak a)

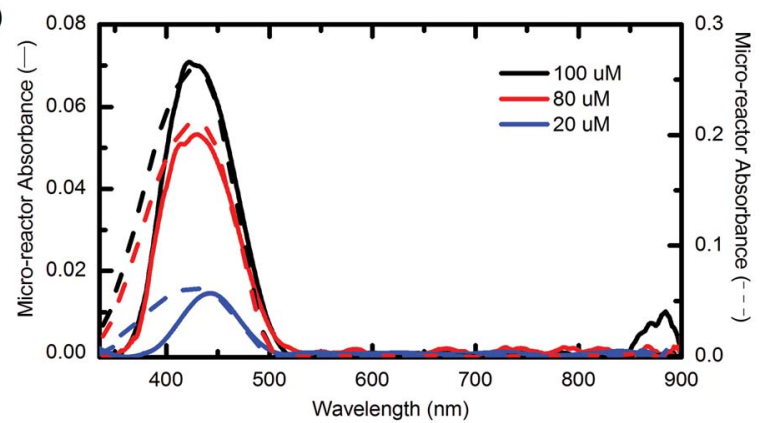

b)

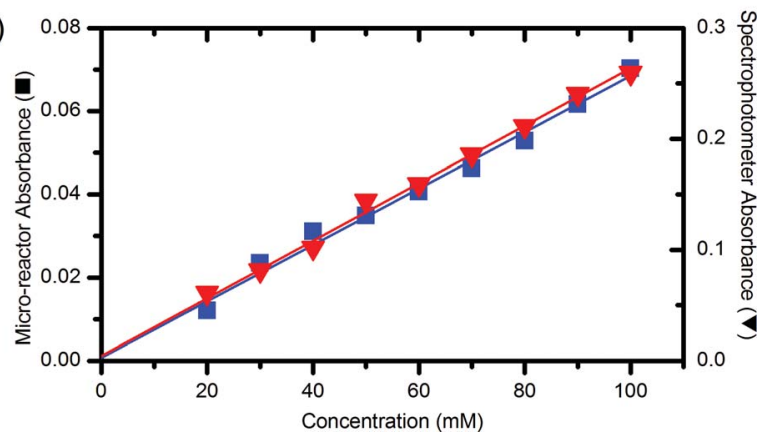

Fig. 2 (a) Absorbance spectra collected with the microscope-coupled spectrophotometer from the microreactor (-), and with the bench spectrophotometer (---). (b) Absorbance of tartrazine solution measured with the microscope-coupled spectrophotometer ( $\square$ ), and with the bench spectrophotometer $(\nabla)$ at a wavelength of $425 \mathrm{~nm}$.

at $425 \mathrm{~nm} .{ }^{4}$ In addition, the limit of detection (LOD) was found to be $20 \mu \mathrm{M}$ for tartrazine solution in the microreactor. For a similar application Waechter et al. ${ }^{5}$ achieved an LOD of $5 \mu \mathrm{M}$, however, with more complex techniques employed including a fiber-loop ring-down spectrophotometer and a diode laser system.

To quantify the system, the extinction coefficient of tartrazine was determined using $\lambda_{\max }=425 \mathrm{~nm}$ (Fig. 2b). The response is linear in the range of $30-100 \mu \mathrm{M}$, and the obtained value of $\varepsilon=2.3 \times 10^{4} \mathrm{M}^{-1} \mathrm{~cm}^{-1}$ agrees well with measurements using a standard spectrometer and with literature values (2.27 $\left.\times 10^{4} \mathrm{M}^{-1} \mathrm{~cm}^{-1}\right){ }^{6}$ The linear correlation between the microreactor and bench-top data is overall excellent. Analogous result were obtained using copper nitrate trihydrate $(10-50 \mathrm{mM})$ at $\lambda_{\max }=809 \mathrm{~nm}$ (ESI $\dagger$ Table S1). Thus the system yields valid numbers over a detection range of at least $400-800 \mathrm{~nm}$. It should be noted that microscope objectives with a significant numerical aperture (NA) could make light rays passing through the sample take on a variety of paths with different angles, and the effective path length taken will differ from the perpendicular value. ${ }^{7}$ This could bring errors in some cases, e.g. with high magnification lenses and inhomogeneous solutions.

Following the validation of the system, we used the setup to monitor the formation progress of silver nanoprisms within the microfluidic reactor, further to our recent studies on microfluidic-based silver nanoprism synthesis, ${ }^{7}$ which were characterised with various off-chip methods including conventional UV-Vis-NIR spectroscopy and TEM. Briefly, solutions were injected with the help of syringe pumps into the microreactor 
with a flow focusing configuration (Fig. 1c), where two reagent solutions were introduced via the two lateral inlets (i.e., $\mathrm{C}_{1}$ and $\mathrm{C}_{3}$ ) while water flowing in from the central inlet (i.e., $\mathrm{C}_{2}$ ). The solutions contained $\mathrm{AgNO}_{3}(36 \mu \mathrm{M})$, trisodium citrate dihydrate (6.5 $\mathrm{mM})$ and $\mathrm{H}_{2} \mathrm{O}_{2}(76 \mu \mathrm{M})$ for injection into $\mathrm{C}_{1}$, and isopropanolic $\mathrm{NaBH}_{4}(3.6 \mathrm{mM})$ into $\mathrm{C}_{3} .{ }^{8}$ The $\mathrm{NaBH}_{4}$ solution was prepared according to Shalom et al. ${ }^{9}$ (see ESI $\dagger$ for details) to avoid hydrogen development in the microchannel which can negatively affect the overall performance of the microreactor. The flow rates of the three streams were optimised to be $1.4 \mathrm{~mL}$ $\mathrm{h}^{-1}$ for both $\mathrm{C}_{1}$ and $\mathrm{C}_{3}$, and $2.4 \mathrm{~mL} \mathrm{~h}^{-1}$ for $\mathrm{C}_{2}{ }^{8}$

It is generally understood that following the mixing of the reagents under these conditions, the formation and growth of silver nanoprisms takes place along the microreactor channel. ${ }^{10}$ Also, the absorbance spectral position $\left(\lambda_{\max }\right)$ and its broadness depend on the size and shape distribution of the nanoparticles. ${ }^{\mathbf{8} 11}$ Therefore, we hypothesised that by monitoring the spectral and absorbance changes at different points along the microreactor channel, the formation process and associated rapid changes in size and shape of silver nanoprisms could be spatially resolved.

Fig. 3c shows the optical absorbance spectra taken from the reaction solution within the microreactor with the incident light beam focused at the middle plane of the microchannel at different positions along the channel. Moving the detector along the channel reveals that particularly between the points at $82.0 \mathrm{~cm}$ and $83.5 \mathrm{~cm}$ (with reference to the channel junction point in the main reaction channel), the optical absorption band shifts from $400 \mathrm{~nm}$ to $750 \mathrm{~nm}$ and becoming significantly broader (Fig. 3c). This spectral change is indicative of the

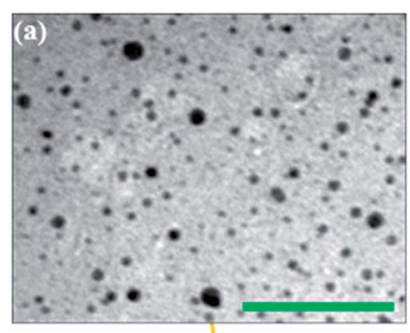

(c)

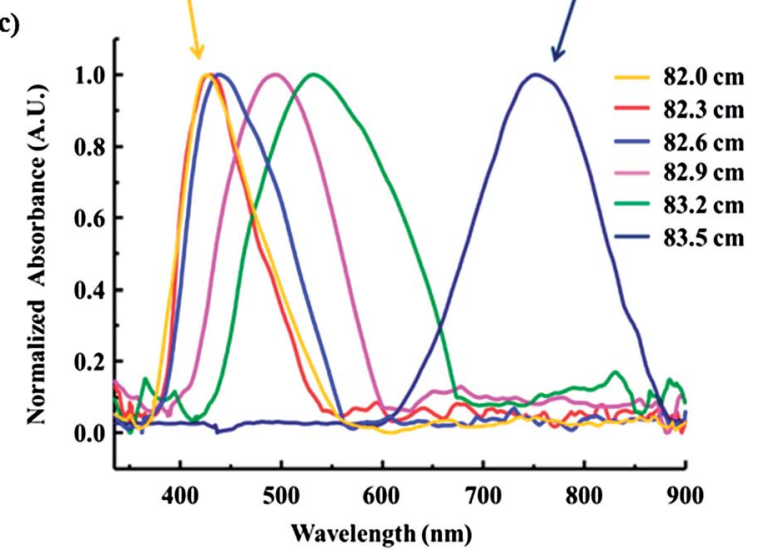

Fig. 3 TEM pictures of (a) silver nanospheres and (b) silver nanoprisms. The scale bars are $100 \mathrm{~nm}$ (a) and $500 \mathrm{~nm}$ (b). (c) Spectral position shift of the absorbance band as a result of detection at different points along the channel. evolution of the nanoparticles from spheres to triangular nanoprisms, which was observed previously in the batch synthesis of nanoprisms. ${ }^{\mathbf{1 2}}$

It is interesting to note that the change occurs in a very short part of the channel, while the spectral properties do not change before or after these points. The assignment of the transition was further confirmed in our study by characterising the nanoparticles with TEM (Fig. 3a \& b). Fig. 3a shows the TEM picture taken from nanoparticles synthesised in a batch reactor exhibiting identical optical properties as in the microchannel up to the $82.0 \mathrm{~cm}$ transition point. Fig. $3 \mathrm{~b}$ shows TEM pictures taken from samples which were collected at the exit of the microreactor. Beyond the point of $83.5 \mathrm{~cm}$ no spectral shifts are observed indicating the formation of stable colloid systems. The long persistence (several minutes) of the yellow solution, indicating spheres, was also observed in batch reactors, followed by a rapid colour change to give prisms. ${ }^{13}$ However, in the microreactor system this process took place in a much shorter time period. The first stage lasted for about $35 \mathrm{~s}$ (i.e., the residence time at the point of $82.0 \mathrm{~cm}$ ) rather than minutes, and rapid change occurred within one second (from the point of $82.0 \mathrm{~cm}$ to $83.5 \mathrm{~cm}$ ). The mechanism for this significantly faster kinetics within the microreactor is unclear, and is under further investigation in our laboratories. It should be noted that for the microfluidic reactor, we found that the use of an aqueous solution of $\mathrm{NaBH}_{4}$ leads to hydrogen gas evolution which has a negative effect on the performance. We therefore use an isopropanolic solution of $\mathrm{NaBH}_{4}$. In our previous studies we also used the isopropanolic solution in a batch reactor. The formation of prisms occurred in reaction times which are comparable to both those reported in the literature as well as to the experimental results obtained in our laboratories using a water solution of $\mathrm{NaBH}_{4}$ (data not shown).

In summary, we have developed a unique microscopecoupled microspectrophotometer for in situ monitoring of reaction evolution within a microfluidic reactor. High quality spectra can be acquired without the need of complex and costly optical setup. Furthermore, collimating the light by use of a custom-made set of lenses allows the light to be focused in small spots, and short integration times are reached along with excellent spatial resolution. The add-on optics have no effects on the use of the microscope, and the system serves well for both spectra and image acquisition. Reducing the fibre diameter, smaller spot sizes can be achieved, which may improve the spatial resolution of the measurements in the channel, however requiring an increase of the integration time (as the light source intensity decreases with smaller fibres). Following validation against conventional spectroscopy, we have demonstrated the application of the system to successfully monitor the shape and size evolution of silver nanoprisms during their formation within a microfluidic reactor. This provides crucial information for further design and control of production of nanoparticles with desired geometries and properties. ${ }^{\mathbf{1 4}}$ The system will be useful for a wider range of spectroscopic measurements, avoiding complex designs of integrated micro optics into the microfluidic devices and dedicated conventional bench-top instrumentations, e.g. that commonly used for studying fast reaction kinetics in stopped- and continuous-flow cells. ${ }^{15,16}$ 


\section{Acknowledgements}

We thank ERDF (ISCE:Chem \& InterReg IVa program 4061) for financial support. We also thank the EPSRC for funding our research (EP/G009732/1 for RZ, and DTC for MC).

\section{Notes and references}

1 R. Salvati, A. Longo, G. Carotenuto, S. De Nicola, G. P. Pepe, L. Nicolais and A. Barone, Appl. Surf. Sci., 2005, 248, 28-31.

2 T. Sounart, P. Safier, J. Voigt, J. Hoyt, D. Tallant, C. Matzke and T. Michalske, Lab Chip, 2007, 7, 908-915.

3 (a) J. Yue, J. C. Schouten and T. A. Nijhuis, Ind. Eng. Chem. Res., 2012, 51, 14583-14609; (b) C. M. Rushworth, J. Davies, J. T. Cabral, P. R. Dolan, J. M. Smith and C. Vallance, Chem. Phys. Lett., 2012, 554, 1-14.

4 R. Jain, M. Bhargava and N. Sharma, Ind. Eng. Chem. Res., 2002, 42, 243-247.

5 H. Waechter, K. Bescherer, C. J. Dürr, R. D. Oleschuk and H.-P. Loock, Anal. Chem., 2009, 81, 9048-9054.

6 H. Waechter, D. Munzke, A. Jang and H.-P. Loock, Anal. Chem., 2011, 83, 2719-2725.
7 I. Broadwell, P. D. I. Fletcher, S. J. Haswell, T. McCreedy and X. L. Zhang, Lab Chip, 2001, 1, 66-71.

8 M. Carboni, L. Capretto, D. Carugo, E. Stulz and X. Zhang, J. Mater. Chem. C, 2013, 1, 7540.

9 D. Shalom, R. C. R. Wootton, R. F. Winkle, B. F. Cottam, R. Vilar, A. J. deMello and C. P. Wilde, Mater. Lett., 2007, 61, 1146-1150.

10 A. Knauer, A. Csáki, F. Möller, C. Hühn, W. Fritzsche and J. M. Köhler, J. Phys. Chem. C, 2012, 116, 9251-9258.

11 D. Aherne, D. M. Ledwith, M. Gara and J. M. Kelly, Adv. Funct. Mater., 2008, 18, 2005-2016.

12 A. Sarkar, S. Kapoor and T. Mukherjee, J. Colloid Interface Sci., 2005, 287, 496-500.

13 G. S. Mètraux and C. A. Mirkin, Adv. Mater., 2005, 17, 412415.

14 I. Shestopalov, J. D. Tice and R. F. Ismagilov, Lab Chip, 2004, 4, 316-321.

15 P. D. I. Fletcher, A. M. Howe and B. H. Robinson, J. Chem. Soc., Faraday Trans. 1, 1987, 83, 985-1006.

16 B. A. Rusin, A. S. Shalomeev, E. K. Russiyan, M. P. Semenovazhukova, A. A. Ivanov and E. M. Brazhnikov, Kinet. Catal., 1979, 20, 246-251. 